\title{
Poster Abstract: Data-driven Comfort Models for User-centric Predictive Control in Smart Buildings
}

\author{
Grigore Stamatescu \\ Automatic Control and \\ Industrial Informatics \\ University "Politehnica" of \\ Bucharest \\ gstamatescu@aii.pub.ro
}

\author{
Alex Beltran \\ Electrical Engineering and \\ Computer Science \\ University of California, \\ Merced \\ abeltran2@ucmerced.edu
}

\author{
Alberto Cerpa \\ Electrical Engineering and \\ Computer Science \\ University of California, \\ Merced \\ acerpa@ucmerced.edu
}

\begin{abstract}
Occupant comfort and energy cost are the dual, explicit or implicit, objectives of advanced control strategies implemented in smart buildings. Focusing on the former, we present a novel approach for data-driven modeling of user comfort and a path for integrating the resulting models in a generic predictive framework for optimal HVAC control. This offers significant improvement potential over the static scheduling or occupancy based temperature bounds.
\end{abstract}

\section{INTRODUCTION}

HVAC systems represent a key focus area to improve efficient energy usage in densely instrumented smart buildings. Conventional control strategies are steadily migrating towards advanced methods such as predictive control which are able to generate optimal, in terms of energy consumption and cost, control inputs to the thermal delivery subsystem over a receding horizon [1]. In terms of occupant comfort, as alternative to standard average assumptions, several systems have been proposed and evaluated, first to collect and subsequently to incorporate occupant feedback regarding the the perceived ambient conditions into the control decision. These typically provide the users with some type of voting mechanism. Long-term experimental evaluations have been presented which illustrate their effectiveness in practice. A common recurring issue is that the occupant voting volume, providing essential comfort feedback to the building operator, abruptly decreases over time due to a variety of reasons which can be related to: lack of interest, decreased novelty factor, comfortable conditions, and others. To mitigate this effect, the paper describes a proposal to handle incomplete and sparse information for filling the gaps in voting patterns at the user and thermal zone levels. The derived comfort model is defined in relation to an s-shaped curve equivalent to a default expected occupant subjective assessment of environmental conditions.

Permission to make digital or hard copies of part or all of this work for personal or classroom use is granted without fee provided that copies are not made or distributed for profit or commercial advantage and that copies bear this notice and the full citation on the first page. Copyrights for third-party components of this work must be honored For all other uses, contact the owner/author(s).

BuildSys '16 November 16-17, 2016, Palo Alto, CA, USA

(C) 2016 Copyright held by the owner/author(s).

ACM ISBN 978-1-4503-4264-3/16/11

DOI: http://dx.doi.org/10.1145/2993422.2996394

\section{DATA-DRIVEN COMFORT MODELING}

A modeling approach is implemented to capture the probability distributions of the user-provided comfort votes, building a generic model. These are subsequently used to enable virtual vote availability for the controller to estimate user comfort at each sampling period and beyond, over the prediction horizon, while the occurence of new real votes enables the update of the model. The raw voting data supporting this study has been collected during multiple experimental deployments, using a system developed in previous work - Thermovote [2]. Vote options follow the 7 point predicted mean vote (PMV) scale, widely used in construction standards and regulations, see ASHRAE 55-2004, and offer the subjective perception of the building occupant in conjuction with a certain timestamp and thermal zone. The scale runs in discrete steps from -3 (very cold) to 3 (very hot), 0 being the ideal comfort condition. Derivation of comfort models is currently performed by aggregating voting data collected between 2015-2016, with the data set consisting of 1500 votes, 45 users and 23 thermal zones. Figure 1a shows the overall voting frequency histogram across symmetric vote and temperature bins within the $60-80 \mathrm{~F}$ interval, while Figure $1 \mathrm{~b}$ illustrates the voting distribution in boxplot format.

The main idea, is to quantify the variance of the voting distribution dataset to a reference empirical comfort model. Fundamental assumption is that the information useful for energy cost savings and overall comfort increase lays in and is captured by the individual and aggregated user numbness/sensitivity to temperature changes. That leads to usercentric control of zone temperatures which takes advantage of two factors: the width of intervals across which the users are insensitive to temperature variations and the steepness of the perceived comfort degradation with various temperature change gradients. Subsequently a reactive system can perform system identification like modeling of the degradation of the comfort conditions for finer grained model estimation. In the current simulation, while acknowledging human factors, we assume engaged users, who offer honest and prompt feedback in regard to their perceived comfort conditions.

As reference, the default comfort model is assumed to take the shape of a S-shaped logistic function:

$$
g\left(T_{z}\right)=\frac{L}{1+e^{-k\left(T_{z}-T_{0}\right)}}
$$

where leveraging the curve parameters $L, k, T_{0}$, empirical local observations and expert knowledge from the facility administrators can be incorporated into the comfort model. Using the available data we quantify the variance from the 


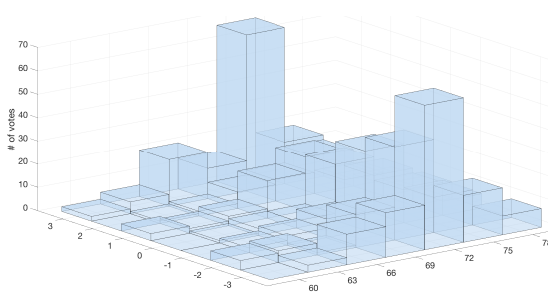

(a) Vote distribution - histogram

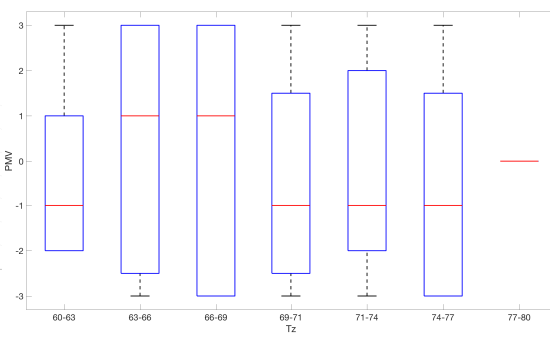

(b) Vote distribution - boxplot

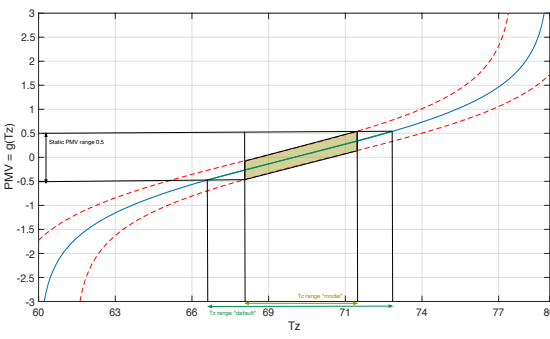

(c) Feasible optimization area

Figure 1: Comfort model enabled MPC

default model and use this as key information in the optimisation framework, defining the allowable margins of the PMV-temperature functional relationship in regard to the default model.

Currently the votes are analyzed in aggregated form, across thermal zones and individual users, in order to observe the high-level tendencies. The method is general such that, with enough votes to define a statistically relevant sample size, we are able to break it down at the zone (weighted model of multiple users) and the individual level.

\section{MPC OPTIMISATION CONSTRAINT}

Given its ability to incorporate the thermal model of the building [3], weather and occupancy forecasts and compute optimal control inputs at each step, over a receding horizon, model-predictive control (MPC) is transitioning towards commercial building management systems. To better accomodate modeling and prediction uncertainty, stochastic approaches represent the state-of-the-art along with hierarchical and distributed architectures for large scale systems e.g. campus-wide collaborative multi-building control [4].

Our formulation of the optimisation problem, to be solved at each sampling step, includes defining the total energy cost objective function to be minimised, subject, along others, to comfort constraints in terms of predicted comfort as it relates to user temperature (absolute and variation) sensitivity. As alternative, the problem can be expressed by jointly minimising cost and comfort through a multiple term objective function or by maximising the comfort with cost constraints. The previous constraint was expressed as:

$$
\bar{T}_{z} \leq T_{z} \leq \underline{T}_{z}
$$

with the lower $\bar{T}_{z}$ and upper $\underline{T}_{z}$ temperature bounds determined by either a static building occupancy schedule or by occupancy estimation in more advanced deployments.

The updated constraint formulation, incorporating the comfort model $g\left(T_{z}\right)$ is as follows:

$$
l b \leq g\left(T_{z}\right) \leq u b
$$

with $g\left(T_{z}\right)$ representing the functional relationship between PMV and zone temperature derived at the modeling stage, and $l b$ and $u b$ being the lower and upper bounds of the comfort interval. Usually the interval would be symmetric around the $g\left(T_{z}\right)=0$ mid-point so that $l b=-u b$. As the interval is statically defined, the building operator can assign various operating modes, depending on the agreed cost-comfort trade-off. Figure 1c illustrates the mapping between the PMV bounds and zone temperature interval as feasible solution region for the optimiser. It is thus argued that by employing this method, improvements in the zone level control inputs might be allowed by adjusting the zone temperature more reliably in correlation to the user's preference as reflected by its past and current voting actions. The building operator also receives increased flexibility in defining the high-level seasonal operating modes. The system is able to improve user perception to the thermal management of the building, by reacting to the feedback that they give, resulting in subjective comfort gains.

\section{CONCLUSIONS AND ONGOING WORK}

The paper discussed an approach to build realistic user comfort models from voting data and include them as tractable optimisation constraints in an MPC framework. The main goal is to achieve HVAC energy cost savings with improved overall occupant satisfaction. Efficient online model update is currently considered i.e. the method of incorporating new votes incoming into the comfort model. The ongoing work is related to the system implementation for extensive experimental evaluation in our campus through a human-in-theloop approach for control of the heating/cooling units.

\section{ACKNOWLEDGMENTS}

This material is based upon work partially supported by the National Science Foundation under grants \#PFI:BIC1430351 and \#CNS-1254192, and the Center for Information Technology Research in the Interest of Society under grant \#2016-0110.

\section{REFERENCES}

[1] G. Stamatescu and A. Cerpa. Zone-level agreement by consensus for building thermal energy management. In 2016 12th IEEE International Conference on Control and Automation (ICCA), pages 377-382, June 2016.

[2] Varick L. Erickson and Alberto E. Cerpa. Thermovote: Participatory sensing for efficient building hvac conditioning. BuildSys '12.

[3] Muddasser Alam and et al. Applying extended kalman filters to adaptive thermal modelling in homes: Poster abstract. In BuildSys '14.

[4] Yushen Long, Shuai Liu, Lihua Xie, and K. H. Johansson. A hierarchical distributed mpc for hvac systems. In 2016 American Control Conference (ACC), July 2016. 